\title{
Accurancy between estimated graft volume and actual graft weight in living donor liver transplant
}

\author{
Ebru H Ayvazoglu Soy ${ }^{1}$, Emre Karakaya ${ }^{1}$, Aydincan Akdur ${ }^{1}$, Gokhan Moray ${ }^{1}$, Mehmet Coskun $^{2}$, Mehmet Haberal $^{1}$ \\ ${ }^{1}$ Department of Surgery, Baskent University, Ankara, Turkey \\ ${ }^{2}$ Department of Radiology, Baskent University, Ankara, Turkey
}

Background: In the living donor liver transplant (LDLT) one of the preoperative evaluation stage is the calculation of graft-recipient-weight ratio (GRWR) and remnant liver volume. According to the Başkent criteria, the remnant volume should be at least $40 \%$ and the GRWR should be at least $1 \%$ in order to minimize postoperative complications and achieve the highest graft functions. We aimed to determine the accuracy of pre-operative computed tomography (CT) graft measurements with actual graft weights in LDLTs in our centers.

Methods: Since 1988, 692 liver transplants have been performed by our team. Of these 692 liver transplants, 480 were LDLTs. Preoperative $\mathrm{CT}$ images and intraoperative graft weights were analyzed retrospectively.

Results: Two hundred seventy (56.3\%) of 480 donors were female and 210 (43.7\%) were male. The mean age of donors was $44 \pm 8.5$ years. The mean weights of donors was $76 \pm 12.5 \mathrm{~kg}$. Of the donor hepatectomies, $47.1 \%(n=226)$ were left hepatectomy, $31.7 \%(n=152)$ right hepatectomy and $21.2 \%(n=102)$ left lateral hepatectomy. The mean total liver volume of donors measured by CT was $1,555.41 \pm 235 \mathrm{~cm}^{3}$. The mean graft volume was $519.41 \pm 250 \mathrm{~cm}^{3}$ and the mean graft weight was $507.37 \pm 242 \mathrm{~cm}^{3}$. When we measured the graft weights during surgery, we found that its ratio to the volume measurements made radiologically was 1.03 . Remnant liver volume in donors was $64.28 \% \pm 15.7 \%$ of the total volume.

Conclusions: Preoperative radiological assessment of the donor's liver is very important to prevent postoperative complications and to perform successfully living related liver transplant. Otherwise, small-for-size or large-for-size may occur in the recipient and liver failure in the donor. 